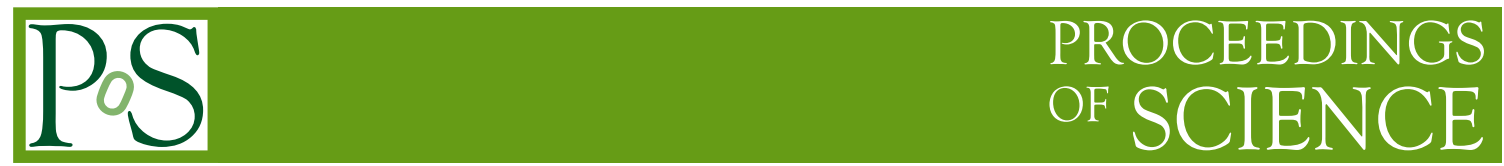

\title{
Open charm and bottom and nonphotonic electrons
}

\author{
Marta Łuszczak* \\ University of Rzeszów, ul. Rejtana $16 c$ \\ PL-35-959 Rzeszów, Poland \\ E-mail: luszczak@univ.rzeszow.pl
}

\section{Antoni Szczurek}

Institute of Nuclear Physics, PL-31-342 Cracow, Poland

and University of Rzeszów, PL-35-959 Rzeszów, Poland

E-mail: Antoni.Szczurek@ifj.edu.pl

\begin{abstract}
We discuss production of nonphotonic electrons in proton-proton scattering at RHIC. The distributions in rapidity and transverse momentum of charm and bottom quarks/antiquarks are calculated in the $k_{t}$-factorization approach. Different unintegrated parton distributions (uPDF) from the literature are used. The hadronization of heavy quarks is done by means of Peterson and Braaten et al. fragmentation functions. The semileptonic decay functions are found by fitting recent semileptonic data obtained by the CLEO and BABAR collaborations. The results are compared with recent results of the PHENIX collaboration.
\end{abstract}

High-pT Physics at LHC - Tokaj'08

16-19 March 2008

Tokaj, Hungary

${ }^{*}$ Speaker. 


\section{Introduction}

Recently the PHENIX collaboration has measured transverse momentum distribution of socalled nonphotonic electrons [1,2]. The dominant contribution to the nonphotonic electrons/positrons comes from the semileptonic decays of charm and beauty mesons. This processes have three subsequent stages. First $c \bar{c}$ or $b \bar{b}$ quarks are obtained assuming gluon-gluon fusion[3] and quarkantiquark annihilation. Next the heavy quarks/antiquarks are turned to heavy charmed mesons $D, D^{*}$ or $B, B^{*}$. The excited $D^{*}$ and $B^{*}$ mesons decay producing $D$ and $B$ mesons. Finally the heavy mesons decay semileptonically producing electrons/positrons. The hadronization of heavy quarks is usually done with the help of fragmentation functions. To obtain the single particle spectra of mesons from those of quarks/antiquarks we used Peterson fragmentation functions [4] and BCFY [5]. The last ingredient are semileptonic decays of heavy mesons. We find the semileptonic decay functions by fitting to recent data of the CLEO [6] and BABAR [7] collaborations. More details about the procedure and more complete presentation of results can be found in our original article [8].

\section{Formalism}

We consider the reaction $h_{1}+h_{2} \rightarrow Q+\bar{Q}+X$, where $Q$ and $\bar{Q}$ are heavy quark and heavy antiquark, respectively.

In the $k_{t}$-factorization approach the cross section in rapidity of $Q\left(y_{1}\right)$, in rapidity of $\bar{Q}\left(y_{2}\right)$ and transverse momentum of $Q\left(p_{1, t}\right)$, transverse momentum of $\bar{Q}\left(p_{2, t}\right)$ can be written as

$$
\begin{array}{r}
\frac{d \sigma}{d y_{1} d y_{2} d^{2} p_{1, t} d^{2} p_{2, t}}=\sum_{i, j} \int \frac{d^{2} \kappa_{1, t}}{\pi} \frac{d^{2} \kappa_{2, t}}{\pi} \frac{1}{16 \pi^{2}\left(x_{1} x_{2} s\right)^{2}} \overline{\left|\mathscr{M}_{i j}\right|^{2}} \\
\delta^{2}\left(\vec{\kappa}_{1, t}+\vec{\kappa}_{2, t}-\vec{p}_{1, t}-\vec{p}_{2, t}\right) f_{i}\left(x_{1}, \kappa_{1, t}^{2}\right) f_{j}\left(x_{2}, \kappa_{2, t}^{2}\right)
\end{array}
$$

where $f_{i}\left(x_{1}, \kappa_{1, t}^{2}\right)$ and $f_{j}\left(x_{2}, \kappa_{2, t}^{2}\right)$ are so-called unintegrated parton distributions.

The production of electrons/positrons is a multi-step process. The whole procedure of electron/positron production can be written in the following schematic way:

$$
\frac{d \sigma^{e}}{d y d^{2} p}=\frac{d \sigma^{Q}}{d y d^{2} p} \otimes D_{Q \rightarrow D} \otimes f_{D \rightarrow e}
$$

where the symbol $\otimes$ denotes a generic convolution. The partonic cross section is calculated in the $k_{t}$-factorization approach. Next step is the process of formation of heavy mesons. We follow a phenomenological approach and take Peterson and Braaten et al. fragmentation functions with parameters from the literature (see e.g. [9]). The electron decay function should account for the proper branching fractions. The latter are known experimentally (see e.g. [6, 7, 9]). These functions can in principle be calculated $[10,11]$. This introduces, however, some model uncertainties and requires inclusion of all final state channels explicitly. An alternative is to use experimental input. The decay functions have been measured only recently $[6,7]$. 


\section{Discussion of the results}

We have calculated inclusive spectra of heavy quarks/antiquarks for RHIC energy in the framework of the $k_{t}$-factorization. We have concentrated on the dominant gluon-gluon fusion mechanism and used two recent unintegrated gluon distribution functions from the literature. In Fig.1 we show for example inclusive cross section for charm/anticharm and bottom production for RHIC energy for the Kwiecinski [13] and Ivanov, Nikolaev [14] unintegrated gluon distributions.
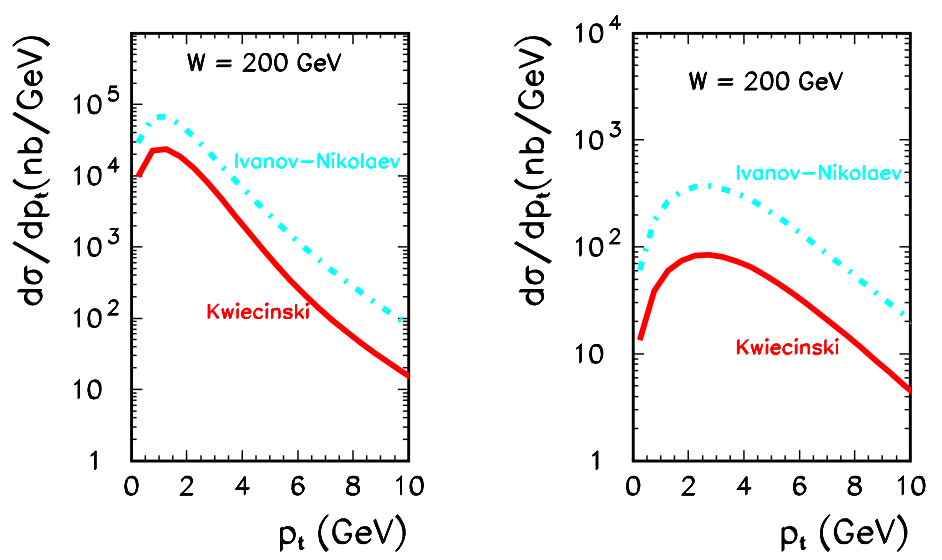

Figure 1: Inclusive cross section for charm/anticharm production (panel a) and bottom production (panel b) for $\mathrm{W}=200 \mathrm{GeV}$ for Kwieciński (solid) and Ivanov-Nikolaev (dashed) UGDF.

In principle, the semileptonic decays can be modeled (see e.g. $[10,12,11])$. Since there are many decay channels with different number of particles this is not an easy task. In our approach we take less ambitious but more pragmatic approach. In Fig.2 we show our purely mathematical fit to not absolutely normalized data of the CLEO [6] and BABAR [7] collaborations.
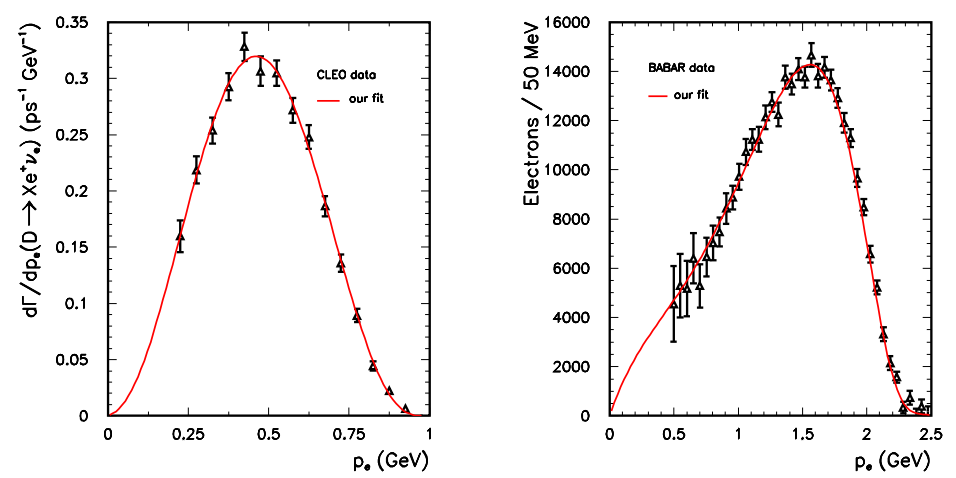

Figure 2: Our fit to the CLEO [6] and BABAR [7] data.

For illustration of the whole procedure in Fig. 3 we show as an example two-dimensional distributions in rapidity and transverse momentum for charm quarks, $D$ mesons and electrons from the 
decay of $D$ mesons. Both fragmentation and semileptonic decays cause degradation of transverse momentum. On average $p_{t, e}<p_{t, D}<p_{t, c}$. The spectra of electrons are much softer than initial spectra of charm quarks. On the other hand the distributions of electrons in rapidity are much broader than the corresponding distributions of quarks/antiquarks.
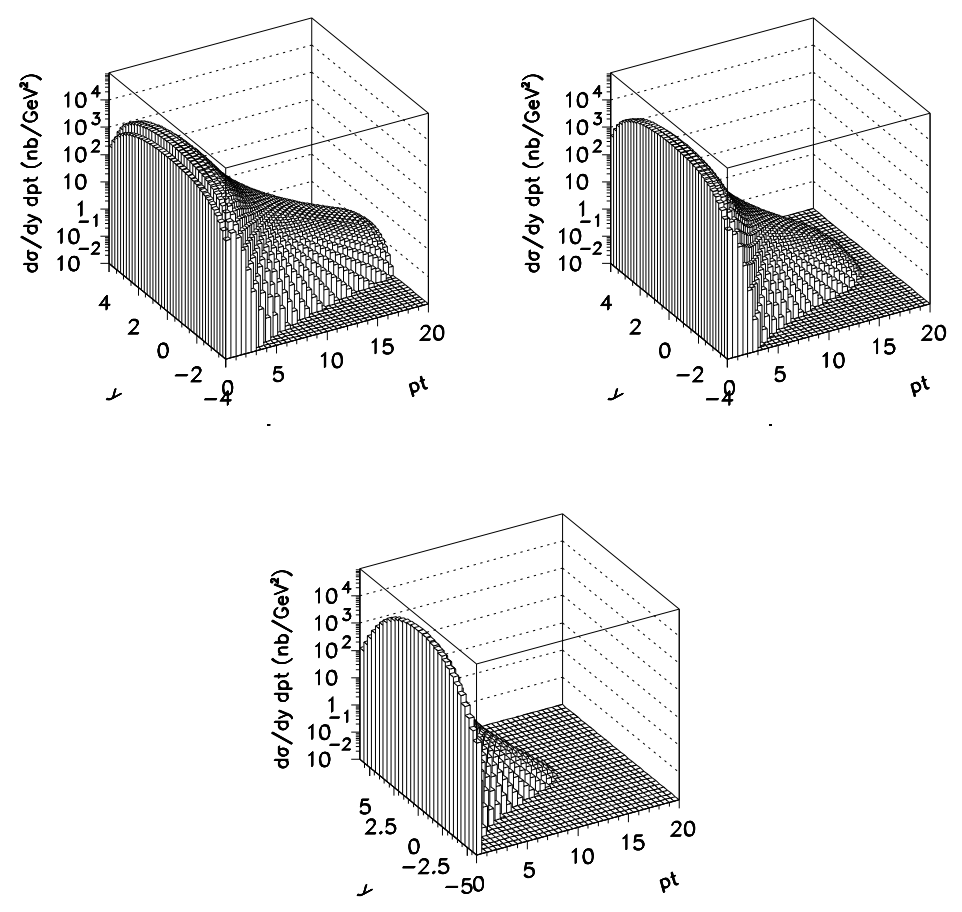

Figure 3: Two-dimensional distributions in rapidity and transverse momentum for charm quark/antiquark, D mesons and electrons/positrons.

We concentrate on invariant cross section as a function of electron/positron transverse momentum. Such distributions have been measured recently by the PHENIX collaboration at RHIC [1]. In Fig.4 we show results obtained with Kwieciński UGDF [13] and different combinations of factorization and renormalization scales as well as for different fragmentation functions (Peterson and BCFY). The differences between results obtained with different combinations quantify theoretical uncertainties. Similarly as for the standard collinear approach [15] one gets uncertainties of the order of a factor 2. We show individual contributions of electrons/positrons initiated by $c / \bar{c}$ or $b / \bar{b}$. The contribution of the $c / \bar{c}$ (dashed) dominates at low transverse momenta of electrons/positrons. At transverse momenta of the order of $4-5 \mathrm{GeV}$ the both contributions become comparable. We obtain rough agreement for large transverse momenta. Similarly as for the higher-order collinear approach [15] there is a missing strenght at lower transverse momenta. A better agreement is obtained with renormalization scale taken as transverse momentum of the initial gluon(s). There are two strong coupling constant in the considered order. In practice we take $\alpha_{s}\left(k_{1 t}^{2}\right) \alpha_{s}\left(k_{2 t}^{2}\right)$, i.e. different argument for each running coupling constant.

In Fig.5 we show results obtained with Ivanov-Nikolaev UGDF. Although there is some im- 

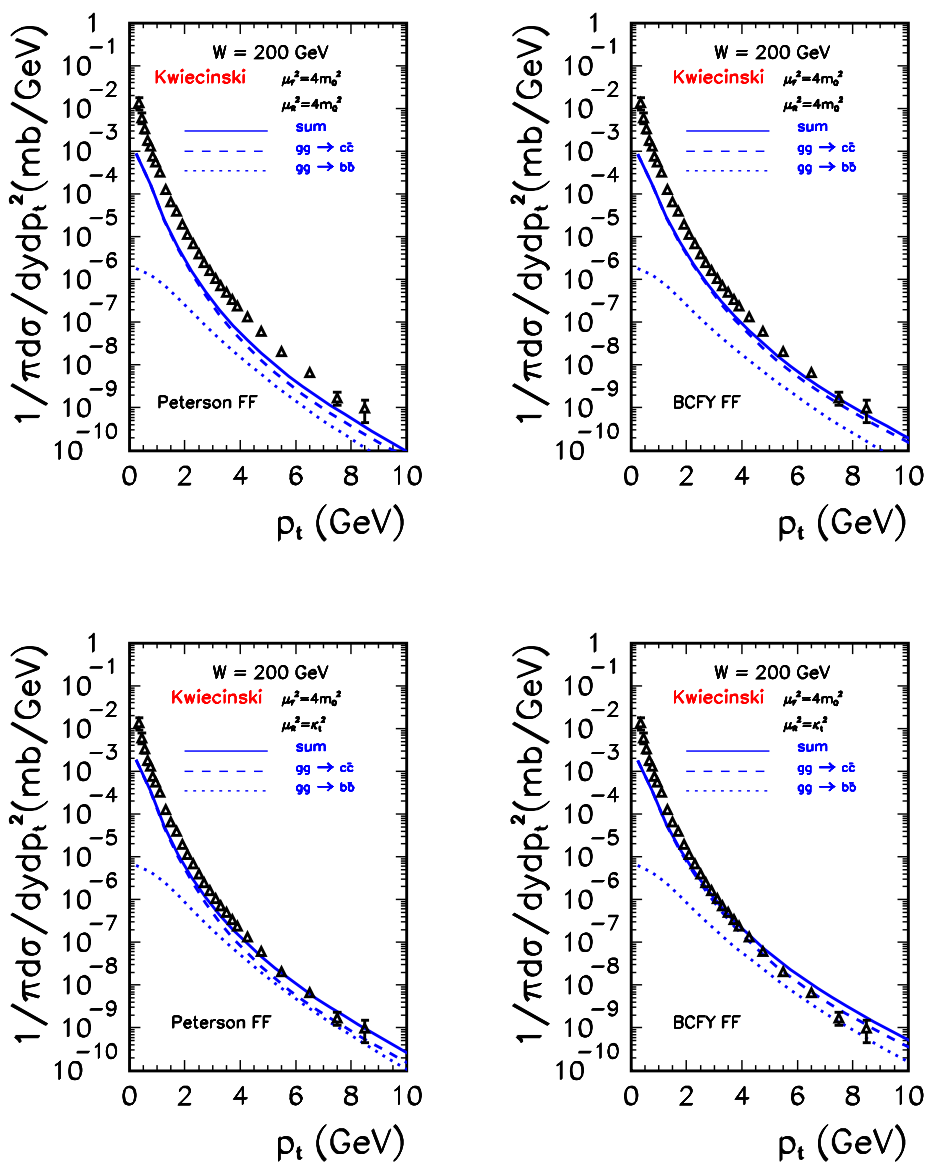

Figure 4: Transverse momentum distribution of electrons/positrons with the Kwieciński UGDF. Different combinations of factorization and renormalization scales are used. On the left side we show results with Peterson fragmentation functions and on the right side with BCFY fragmentation functions.

provement at low transverse momenta, the cross section for larger transverse momenta exceeds the experimental data.

It is not clear for the moment what is the missing strength. Up to now we have included only gluon-gluon fusion which is known to be dominant contribution at large center-of-mass energies. The RHIC energy is, however, not too high. Therefore we shall try to include also quark-antiquark annihilation process. Those processes can be included in a similar way in the formalism of unintegrated parton distributions.

The Kwieciński formalism [13] allows to calculate unintegrated quark/antiquark distribution in the same framework as unintegrated gluon distributions. In Fig.6 we present the contribution of quark-antiquark annihilation $q \bar{q} \rightarrow c \bar{c}$ (dash-dotted line). This contribution is similar in size to the $g g \rightarrow b \bar{b}$ contribution. The contribution of $q \bar{q} \rightarrow b \bar{b}$ is negligible and is not shown here. 

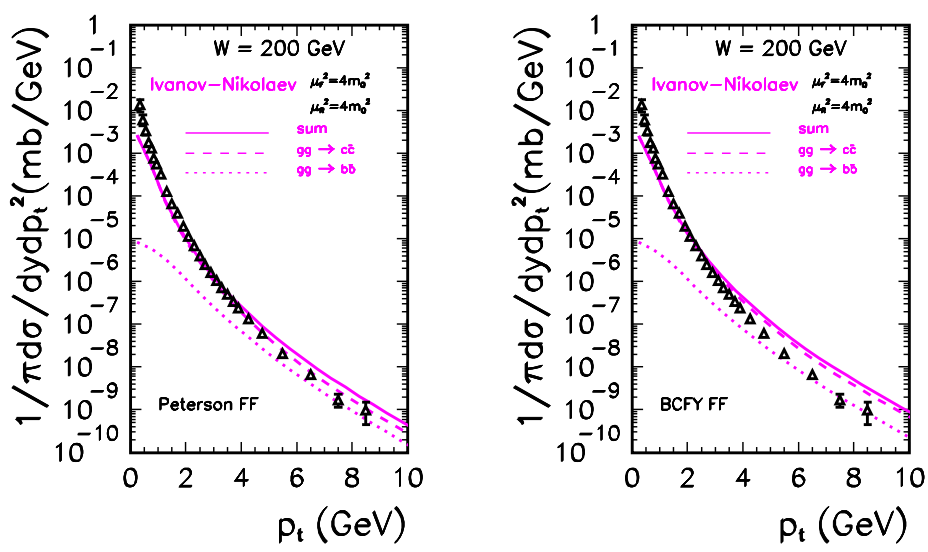

Figure 5: Transverse momentum distribution of electrons/positrons obtained with Ivanov-Nikolaev UGDF and Peterson (left panel) and BCFY (right panel) fragmentation functions.

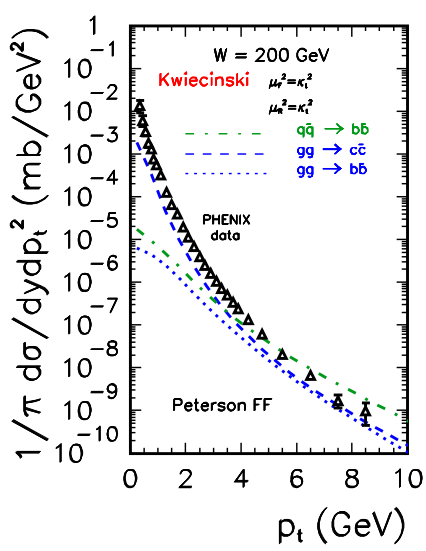

Figure 6: Transverse momentum distribution of electrons/positrons with Kwiecinski UPDFs. The dashdotted line corresponds to the $q \bar{q} \rightarrow b \bar{b}$ contribution.

\section{References}

[1] A. Adare et al., Phys. Rev. Lett. 97 (2006) 252002, arXiv:hep-ex/0609010.

[2] J. Adams et al., Phys. Rev. Lett. 94 (2005) 062301.

[3] M. Łuszczak and A. Szczurek, Phys. Rev. D73 (2006) 054028.

[4] C. Peterson, D. Schlatter, I. Schmitt, P.M. Zerwas, Phys. Rev. D27 (1983) 105.

[5] E. Braaten, K. Cheung, S. Fleming and T.C. Yuan, Phys. Rev. D51 (1995) 4819.

[6] N.E. Adam, et al. (CLEO collaboration), Phys. Rev. Lett. 97 (2006) 251801, hep-ex/0604044.

[7] B. Aubert, et al. (BABAR collaboration), Phys. Rev. D69 (2004) 111104(R). 
[8] M. Łuszczak, R. Maciuła and A. Szczurek, arXiv:0807.5044.

[9] W.M. Yao et al., [Partilce Data Group], J. Phys. G33 (2006) 1.

[10] R. J. Hill, arXiv:hep-ph/0606023.

[11] M. Artuso, B. Meadows, A. A. Petrov, arXiv:0802.2934.

[12] H. Mahlke, arXiv:hep-ex/0702014.

[13] J. Kwieciński, Acta Phys. Polon. B33 (2002) 1809. A. Gawron and J. Kwieciński, Acta Phys. Polon. B34 (2003) 133. A. Gawron, J. Kwieciński and W. Broniowski, Phys. Rev. D68 (2003) 054001.

[14] I.P. Ivanov and N.N. Nikolaev, Phys. Rev. D65 (2002) 054004.

[15] M. Cacciari, P. Nason and R. Vogt, Phys. Rev. Lett. 95 (2005) 122001. 